\title{
Modeling of heat transfer in a hot bath
}

\author{
Liwen Peng \\ School of North China Electric Power University, Hebei 071003, China \\ 2537531989@qq.com.
}

Keyword : Goal programming Heat transfer theory Newton cooling formula

\begin{abstract}
In this paper, we build the model to determine the best strategy that the person in the bathtub can keep the temperature.

Firstly, we assume that the bathtub is a fixed size water containment vessel. In case of no external heat source, we set up a water temperature model of the bathtub by using the knowledge of heat transfer, get the figure of temperature change with time.

Secondly, adding a single faucet which can inject hot water continuously keeps the temperature of the water in the bathtub. Let the heat of the hot water equal to the loss of heat,obtaining the relationship between the temperature and the flow rate of the hot water. By listing the target of water-saving amount, constrained conditions of temperature, constrained conditions of water consumption, and set up the goal programming model. Using MATLAB to solve the model, we obtain the best strategy that the minimum water consumption is 2016L,temperature of hot water injected is $54.5^{\circ} \mathrm{C}$, and the flow rate is $0.28 \mathrm{~L} / \mathrm{s}$.
\end{abstract}

\section{1、 Introduction}

We build a Heat transfer model of the temperature of the bathtub water in time.Most importantly, the model reflect the heat loss of the bath under the natural conditions Then,we add a constant trickle of hot water from the faucet to reheat the bathing water, the flow and the temperature are constant, and let the heat quantity of faucet injection is equal to the heat loss. Finally, we can obtain the relationship between the flow rate and the temperature of hot water injection.

\section{2、The Model of temperature changing with times}

To simplify the problem, we make the following basic assumptions, each of which is properly justified.Firstly, the Heat loss from the faucet to the surface of the water is ignored.Secondly, assume that the temperature distribution of the wall of the bathtub is uniform.

The model is based on the knowledge of heat transfer. Assume that the bath size is $1500 \times 800 \times 400 \mathrm{~mm}$, the water temperature is $40^{\circ} \mathrm{C}$ and the bathroom air is $30^{\circ} \mathrm{C}$. When calculating the loss of heat, we mainly consider three aspects as follows.

One from the surface of the water to the air,one from the water to the bathtub to the air again the wall and another from the water to the bathtub again the ground.

\subsection{The loss of heat from the surface of the water to the air}

Convection heat transfer ${ }^{[1]} d q_{a}$, evaporation ${ }^{[2]}$ dissipation $d q_{b}$, thermal radiation $d q_{c}$. Therefore, the total heat transfer rate from the water surface to the air is:

$$
q_{1}=\int d q_{a}+d q_{b}+d q_{c}
$$

\subsection{The loss of heat ${ }^{[3]}$ from the water to the bathtub to the air again the wall.}

The heat Circuit Diagram as follow : 


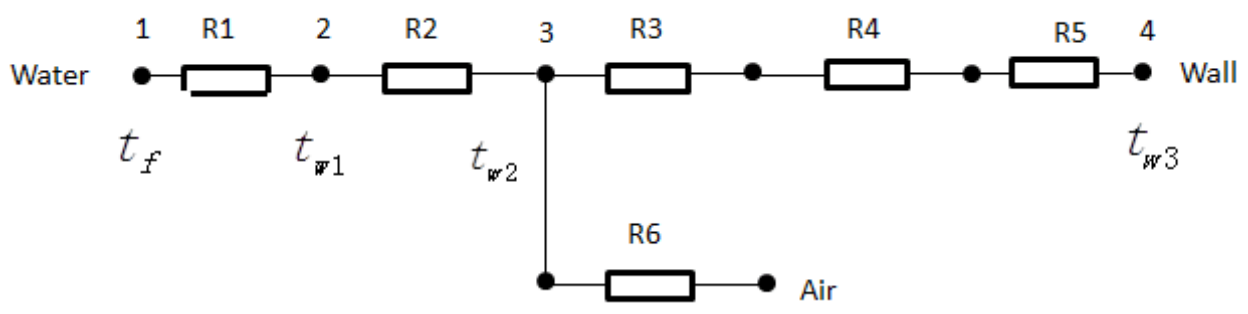

Figure 1 Heat transfer 1

The heat flow of total heat dissipation in this direction is :

$$
q_{2}=\frac{t_{f}-t_{w 2}}{R_{1}+R_{2}+R_{3}}
$$

\subsection{The loss of heat from the water to the bathtub again the ground.}

The heat Circuit Diagram as follow:

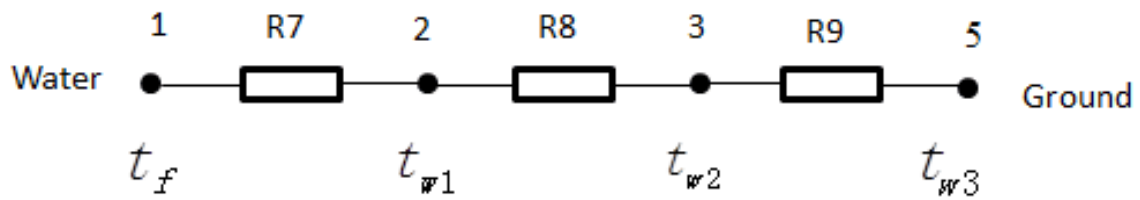

Figure 2 Heat transfer 2

Therefore, the heat flow of total heat dissipation in this direction is :

$$
q_{3}=\frac{t_{f}-t_{w 3}}{R_{7}+R_{8}+R_{9}}
$$

Above all , the total heat flow of bath water loss per unit time under natural conditions is : $q=q_{1}+q_{2}+q_{3}$

In order to keep the temperature as close as possible to the initial temperature now, the person adds a constant trickle of hot water from the faucet to reheat the bathing water. The heat flow of injected hot water is equal to the total amount of the amount of heat dissipation : $q_{w}=q$

$$
q_{w}=p_{m} \times C \times \Delta t=q_{v} \times \rho \times C \times\left(t_{d}-t\right)
$$

Where $q_{v}$ is the volume flow, $\rho$ is the density of water, $C$ is the specific heat capacity of water. $t_{d}$ is the injected hot water temperature.

$$
q_{v} \times \rho \times C_{v} \times\left(t_{d}-40\right)=18900
$$

We assume that the bath time more than one hour, the bath is in an overflow state in most of the time Therefore, we mainly consider the constant temperature strategy in an overflow state.Because the average temperature in the bath will be smaller than the final constant temperature at least 2 degrees, and according to Newton cooling formula, we assume that $40 \leq t_{d} \leq 60$. We list the targets of water-saving amount, the constraint conditions of temperature, the constraint conditions of the water consumption and set up a goal programming model.

$$
\begin{aligned}
\min V=q_{v} t \\
\mathrm{s.t}: \quad q_{v} \times \rho \times C_{v} \times\left(t_{d}-40\right)=18900 \\
\rho \times C \times V \times \frac{d t}{d \tau}=-h A(t-30) \\
-\frac{\sum t_{i j k}}{150 \times 80 \times 40} \\
\mid-\frac{-}{t-40) \mid<2}
\end{aligned}
$$




$$
40 \leq t_{d} \leq 60
$$

The best strategy of using MATLAB that $q_{v}=0.28 \mathrm{~L} / \mathrm{s}, t_{d}=54.5^{\circ} \mathrm{C} \tau=167 \mathrm{~s}$, We get the minimum water consumption that $V=2016 \mathrm{~L}$.

\section{Summary}

According to the model, we can use the same method to find the best water saving strategies for different shapes of bath.

In the hypothesis, we ignore the hot water, $\mathrm{s}$ heat loss from the faucet to the bath level .This will result in a reduction in the quantity of heat, which makes the analysis result in a certain error. If we consider this loss, we will get a more accurate result of the strategy by further modification of the model.

\section{References}

[1] Zhenguo Zhao, Formula and application of baking difference of surface heat radiation[J],Journal of hydraulic engineering, 2004.

[2] Shiming Mao,Huiquan Chen, Discussion on the formula and application of heat

dissipation of water[J], Journal of hydraulic engineering,2004.

[3] Rudolph G.Local convergence rates of simple evolutionary algorithms with Cauchy mutations.IEEE Transaction Evolutionnary Computation,1997.

[4] YUNUS A CENGEL.Heat transfer,A Practical Approach. New York;McGraw-Hill Book Company,2003. 